\title{
Erratum to: Radiofrequency versus ultrasonic energy in laparoscopic colorectal surgery: a metaanalysis of operative time and blood loss
}

Nicola Di Lorenzo • Luana Franceschilli •

Marco Ettore Allaix • Anastasios Asimakopoulos •

Pierpaolo Sileri $\cdot$ Achille L. Gaspari

Published online: 14 December 2012

(C) Springer Science+Business Media New York 2012

Erratum to: Surg Endosc (2012) 26:2917-2924

DOI 10.1007/s00464-012-2285-5

The first author's name was misspelled as N. D. Lorenzo.

The correct spelling is N. Di Lorenzo.

The online version of the original article can be found under doi:10.1007/s00464-012-2285-5.

N. Di Lorenzo $(\bowtie) \cdot$ L. Franceschilli · P. Sileri · A. L. Gaspari Department of Surgical Sciences, University of Rome Tor Vergata, Policlinico Tor Vergata, Viale Oxford 81, 00133 Rome, Italy

e-mail: Nicola.di.lorenzo@uniroma2.it

L. Franceschilli

e-mail: luana.franceschilli@virgilio.it

N. Di Lorenzo · L. Franceschilli · M. E. Allaix ·

A. Asimakopoulos · P. Sileri · A. L. Gaspari

Biotechnologies and Techniques Applied to Surgical Sciences,

University of Rome, Tor Vergata, Italy

M. E. Allaix

Department of Digestive Surgery and Centre for Minimally Invasive Surgery, University of Turin, Turin, Italy

A. Asimakopoulos

Division of Urology, Department of Surgical Sciences,

University of Rome, Tor Vergata, Italy 